\title{
Kawa-COVID-19 Storm: Multisystem inflammatory syndrome in children (MIS-C) associated with SARS-CoV-2?
}

\author{
Marcos Altable
}

Funding: The author(s) received no specific funding for this work.

Potential competing interests: The author(s) declared that no potential competing interests exist.

\begin{abstract}
Multisystem inflammatory syndrome in children (MIS-C) is a new type of serious disease that affects children in childhood and is associated with infection with SARS-CoV-2.

According to current data, COVID-19 is less common in children and has a milder course. Recently, however, there has been an increase in the number of pediatric patients worldwide who have symptoms resembling Kawasaki disease after overcoming COVID-19 infection. Kawa-COVID-19 probably represents a new systemic inflammatory syndrome associated with SARS-CoV-2 (MIS-C) in children. Symptoms of Kawa-COVID-19 occur in pediatric patients approximately two weeks after SARS-CoV-2 infecton, and in $50 \%$ of cases, the most common complication is myocarditis. Prognostic severe factors include patients over five years old and ferritin concentrations> $1400 \mu \mathrm{g} / \mathrm{L}$. Further international studies will be needed in the future to help better understand the nature of the disease.
\end{abstract}

\section{Introduction}

Since the first reported case of pneumonia in December 2019 in Wuhan, China, caused by a novel coronary virus (COVID19) with severe acute respiratory syndrome (SARS-CoV-2), a pandemic has occurred with more than 200,000 deaths worldwide $(1,2)$. Unlike adults, children are less susceptible to the disease, and the course is milder (3).

The number of SARS-CoV-2 cases has reached over 19.8 million confirmed cases, and 730,000 deaths. Studies have 
noted that children with COVID-19 are safe from developing the infection, as they suffer from simple symptoms in the upper respiratory system, as one study confirmed that out of 171 children infected with COVID-19, only 3 children are required to enter into intensive care, with one death recorded. But in 2020, new symptoms appeared on children with COVID-19, including a high temperature, a rash, conjunctivitis and pain in the digestive system. It was diagnosed by Royal College of Paediatrics and Child Health (RCPCH) as a pediatric multisystem inflammatory syndrome temporally associated with COVID-19 (MIS-C) (46).

The most important challenge was the great similarity between MIS-C versus Kawasaki disease (KD) or toxic shock syndrome (TSS). Kawasaki disease (KD) is an inflammatory blood vessel disease associated with a high temperature in children less than 5 years old. Although the disease may appear mild, it may reach a dangerous stage if inflammation occurs in the coronary artery, or in the heart muscle, or by the occurrence of strokes (47).

Kawasaki disease can be divided into classic or incomplete based on the following criteria:

Fever of more than 5 days with 4 or more of the following criteria: conjunctival injection, fever, edema of hands and feet, lymphadenopathy, inflammation of the oral membranes. In contrast, an incomplete KD includes diagnosis if the fever lasts 2-3 days (48).

$\mathrm{KD}$ is an acute systemic vasculitis of unknown aetiology that affects medium-calibre arteries and leads to the development of coronary artery aneurysms in approximately $25 \%$ to $40 \%$ of untreated patients. It occurs mainly in children under the age of five. KD is the leading cause of acquired heart disease in pediatric patients in developed countries (10). Pericarditis occurs in $18 \%$ of patients. Myocarditis occurs in $3 \%$ of cases and occasionally causes cardiogenic shock in the acute stage of the disease $(11,12)$. The risk of developing coronary artery aneurysms is reduced in patients treated with intravenous immunoglobulins (IVIG) to approximately $3-6 \%$. Seasonal variations, epidemiological data, and the low risk of disease recurrence suggest that several infectious agents may be an essential trigger for KD, although no specific microorganisms have been identified to date (13). Genetic factors may be involved in the pathophysiology of KD, which leads to stimulation and activation of the innate immune system $(15,16)$.

In addition to the cases mentioned above in the US and UK, a group of doctors from France also described 16 cases of COVID-19 / SARS-CoV-2 patients between March and April 2020 who developed the disease atypically form of KD (17). The main purpose of this review is to clarify the vision for doctors and therapists for ease of diagnosis and to take appropriate decisions in the treatment strategy, which contributes to raising the cases of recovery.

\section{Main}

Until recently, COVID-19 was considered an almost harmless disease in children (2). Pouletty et al. describe a group of 16 patients with a KD-mimicking systemic inflammatory syndrome associated with SARS-CoV-2 (Kawa-COVID-19) infection. This severe disease that required intensive care was observed in $44 \%$ of the cases. All patients presented complete or incomplete symptoms of KD, but there are differences that quite clearly reflect the difference between the typical form of KD and Kawa-COVID-19 and suggest that this disease represents a new entity also confirmed by the study of Verdoni et al. First, the median age of pediatric patients is higher than in classic KD (> 5 years). Second, the frequency and severity of myocarditis are strikingly different and higher than with the classic form of KD. Third, abdominal pain and diarrhoea are much more common symptoms (almost $81 \%$ of patients) than in classic KD (approximately one in three patients) (18). 
Finally, heart failure, pneumonia, gastrointestinal, nervous, and kidney disorders, elevated C-reactive protein (CRP), ferritin, and cytokines (especially IL-1, TNF- $\alpha$, and IL-6) are more common in Kawa-COVID-19. In addition to heart failure, these symptoms are also common in adult patients with severe COVID-19 19, 20. However, unlike adults, pediatric patients with Kawa-COVID-19 rarely show respiratory symptoms, suggesting a different immune response in children. However, in almost everything in pediatric patients without respiratory symptoms, diffuse peripheral vitreous lesions were observed in the lungs bilaterally on computed tomography (17).

In light of the above, paediatricians should suspect Kawa-COVID-19 in any child with an unexplained fever lasting more than four days and elevated CRP in the context of a pandemic, especially if any of the clinical signs of COVID-19 are present or comes in contact with is confirmed with an infected patient during the last month. In such cases, the classic clinical signs of KD and symptoms of the respiratory system or gastrointestinal tract should be closely monitored. As part of prevention, some tests should be done routinely to diagnose life-threatening complications. These include the diagnosis of myocarditis (troponin, natriuretic peptide - NT-proBNP, ECG), macrophage activation syndrome (white blood cells, fibrinogen, ferritin, and albuminuria) and renal failure (creatinine, urea, and proteinuria). Doctors should be especially careful with patients older than five years with elevated ferritin levels (above 1,400 $\mu \mathrm{g} / \mathrm{L}$ ) due to the risk of rapid progressive Kawa-COVID-19 heart failure (17).

Patients should be evaluated by RT-PCR for SARS-CoV-2 (reverse transcription-polymerase chain reaction), at least from nasopharyngeal secretions or stool samples. Serological tests are also recommended (they may be negative due to the late onset of this syndrome). Additional virologic examinations should rule out alternative diagnoses.

The study by Pouletta et al. demonstrates a direct link between KD and SARS-CoV-2. Interestingly, all these cases occurred between April 7 and April 30, 2020, one week after the epidemic outbreak in the Greater Paris region between March 31 and April 1, 2020. There has also been an increase in atypical forms of KD in other countries, including the US (17).

The Royal College of Pediatrics and Child Health (UK) and the New York City Department of Health (US) issued guidelines in late April 2020 and early May 2020 on associated childhood multisystemic inflammatory syndrome with COVID-19. Furthermore, information on hyperinflammatory shock in a group of children during COVID-19 treatment has been published recently $(6,8,9,20)$.

\section{Pathophysiology of KS and Kawa-COVID-19}

Like some viral diseases, KS is a high fever illness with rash, swollen lymph nodes, enanthemum, and conjunctival injection. Scarlet fever should be mentioned as a bacterial disease with clinically similar symptoms. Despite these similarities, it has not yet been possible to detect germs, and a large number of infectious agents are discussed as causes. The cause was suspected to be retrovirus, adenovirus, EBV, human herpesvirus 6, chlamydia, streptococci, mycobacteria, and house dust mites.

There are several contradictory hypotheses about the aetiology and pathogenesis of Kawasaki syndrome, which is why the disease classification is controversially discussed.

One hypothesis views KS as a disease in which an infectious agent triggers a prolonged, self-directed immune response. The KS would be between infectious disease and autoimmune disease. According to this model, among other things, a 
superantigen is supposed to activate the immune system. This assumption is based, among other things, on a KS mouse model in which the induction of coronary arteritis is directly correlated with the superantigen activity of the Lactobacillus casei cell wall extract. This superantigen triggers the local production of IFN- $\gamma$ and TNF- $\alpha$ in the walls of the affected vessels; TNF- $\alpha$ is of central importance for inflammation of the vessels, mice with the inactive TNFR1 gene do not get sick. A special feature of the Lactobacillus superantigen is protecting activated T cells against apoptosis through increased costimulation. This is mediated by up-regulation of TLR-2 (toll-like receptor) and results in the production of MMP-9 (matrix metalloproteinase), a molecule that is essentially responsible for aneurysm formation (22-24), and it has been implicated in COVID-19 (25).

Another theory relies on a ubiquitous and previously unknown RNA virus as the trigger. This is supported by the following points: although neutrophils are the predominant cell type in the blood of KS patients, mainly mononuclear cells are found in the walls of inflamed vessels, and CD8 + T cells predominate in T cells. IgA plasma cells are dominant among immunoglobulin-producing cells. The combination of T cells CD8 + and IgA plasma cells suggests an immune response against an intracellular pathogen. The $\lg A$ response is oligoclonal, and synthetic $\lg A$ antibodies can be used to detect a specific antigen on the bronchial epithelium and macrophages of inflamed KS tissue. This antigen resides in cytoplasmic inclusion bodies that contain a viral protein and ribonucleic acids.

According to this hypothesis, KD is caused by a still unknown "new" RNA virus that infects the bronchial epithelium. This infection remains asymptomatic in non-susceptible children but triggers the disease in a group of genetically susceptible children (26).

Another complementary hypothesis was proposed by Rodó et al. proposes. After that, tropospheric winds are said to bring the causative agent of Kawasaki disease from northeast China to Japan. The postulated windborne agent could be related to several hypotheses mentioned above. However, the authors themselves later pointed out the complexity and open questions of their "wind hypothesis" $(27,28)$.

Regardless of the infectious cause that leads to the disease, there is undoubtedly a genetic predisposition in KS. This fact is suggested, among other things, by the much higher incidence of the disease in Japanese children, which remains higher even if they live in the United States. Consequently, genome-wide association studies have shown several genes associated with the risk of developing KS. Other loci are associated with the risk of cardiovascular disease or resistance to immunoglobulin therapy $(24,29,30)$.

KS leads to systemic activation of the immune system. This is demonstrated by the increase of various cytokines IL-1, TNF- $\alpha$, IFN- $\gamma$, IL-4, IL-6, IL-8 and IL - 10. Cell activation markers and adhesion molecules such as ICAM-1, VEGF, PDGF, and CD84 are also increasingly expressed. This triggers the inflammation of the vessels. These results show that both the nonspecific and specific immune systems are involved in the pathogenesis and suggest a process triggered by an antigen $(26,31)$.

The pathophysiology of Kawa-COVID-19 has yet to be clarified. Post-infectious processes with delayed activation of the immune system, leading to a cytokine storm, are responsible for fever, skin symptoms, heart failure, and inflammatory syndrome. This mechanism appears to be a possible presumed pathophysiology of atypical KD (32-34). Kawa-COVID-19 develops 2-4 weeks after overcoming acute SARS-CoV-2 infection. In some patients, residual faecal virus shedding was demonstrated in a negative nasopharyngeal test. Nevertheless, all the patients had positive lgG 
antibodies, confirming a recent infection (2 to 4 weeks) with SARS-CoV-2 $(35,36)$. These findings indirectly indicate that the consequences of a previous SARS-CoV-2 infection may act as a trigger and cause additional inflammatory disease. Viral elements have also been found in endothelial cells of patients infected with SARS-CoV-2, as well as signs of accumulation of inflammatory changes, apoptosis, and pyroptosis (37). Damage to endothelial cells may explain subsequent autoinflammatory changes (vasculitis) with activation of IL-1 or IL-6 $(38,39)$.

The nonspecific immune response that pediatric Kawa-COVID-19 patients have compared to adults remains questionable and the presence of specific genetic factors that may predispose children to more than adults in response to inflammatory disease.

\section{Treatment}

Patients should be closely monitored and managed therapeutically for the rapid and progressive development of the disease.

Previous studies presented pediatric patients with cardiovascular complications, either myocarditis or coronary artery aneurysms (44\% vs. $19 \%)(12,40)$.

According to American Heart Association (AHA) guidelines, an IVIG injection ( $2 \mathrm{~g} / \mathrm{kg}$ ) should be given with aspirin while the patient is feverish. It has been suggested that glucocorticoids should be given to patients with possible coronary artery aneurysms (49).

In some treatment centers, patients with MIS-C were considered to be of high risk, so they should be given IVIG, aspirin and pulse steroids. One study found that (11\%) of patients treated with IVIG had a relapse, so in these cases they resort to giving them biological treatment such as anti- IL-6, anti-TNF (50).

Since most patients with MIS-C are prone to vascular thrombosis, they should be given anticoagulants in the treatment protocol (51).

However, $31 \%$ of the patients required treatment with anti-inflammatory steroids. Complementary anti-inflammatory therapy should be considered in patients with severe disease or the presence of prognostic factors, especially those over 5 years of age and high levels of ferritin (> $1400 \mu \mathrm{g} / \mathrm{I})(17)$.

According to the literature, some other methods of treating KD are being considered. Steroids have been used successfully in KD therapy for several years. Anakinra, which acts as an IL-1 receptor antagonist, shows promise. Attempts have been made to use this drug in cases of refractory disease (40), and its strong anti-inflammatory effects are still under investigation in clinical studies (41) (KAWAKINRA, Clinical Trials. Gov.: NCT02390596).

Tocilizumab (an anti-IL-6 receptor monoclonal antibody) is used to treat juvenile idiopathic arthritis since their symptoms are reminiscent of Kawa-COVID-19 symptoms: rash, fever, arthritis, multisystem inflammatory syndrome, elevated ferritin levels, etc. Patients with severe COVID-19 have elevated IL-6 levels, and treatment with tocilizumab has shown promising results (CORIMUNO-19, Clinical Trials. Gov.: NCT04331808) 42, 43. In the framework of international cooperation, more multicenter studies are needed to evaluate the efficacy of these drugs.

\section{Conclusion}


Kawa-COVID-19 is a systemic inflammatory disease that resembles KD and is associated with SARS-CoV-2 infection. However, this new disease differs from classic KD in that it occurs in older children and has a higher frequency of complications such as severe myocarditis or pericarditis. Prognostically unfavourable outcome criteria in pediatric patients include age older than 5 years and elevated ferritin levels (> $1400 \mu \mathrm{g} / \mathrm{I})$. Single IVIg administration in these studies resulted in remission of inflammatory changes in only $31 \%$ of patients, and $62 \%$ required a second line of treatment. Influenza vaccination and rational antibiotic therapy remain essential during the respiratory season $(44,45)$. Another challenge in international cooperation is elucidating the immune response and genetic factors better to understand the pathophysiology and entity of this emerging disease.

Despite the physiological similarities between MIS-C and Kawasaki disease, there are some differences that can explain the difference between them. Also, reports indicate that children with MIS-C who have been infected with Covid 19 are rapidly deteriorating, so they need careful care, and the condition may reach the need for intensive care.

\section{References}

1. Cohen JF, Korevaar DA, Matczak S, Chalumeau M, Allali S, Toubiana J. COVID-19-Related Fatalities and Intensive-Care-Unit Admissions by Age Groups in Europe: A Meta-Analysis. Front Med. 2021;7. doi:10.3389/fmed.2020.560685

2. Dong E, Du H, Gardner L. An interactive web-based dashboard to track COVID-19 in real time. Lancet Infect Dis. 2020;20(5):533-534. doi:10.1016/S1473-3099(20)30120-1

3. Lu X, Zhang L, Du H, et al. SARS-CoV-2 infection in children. N Engl J Med. 2020;382(17):1663-1665.

4. Parri N, Lenge M, Buonsenso D. Children with Covid-19 in Pediatric Emergency Departments in Italy. N Engl J Med. 2020;383(2):187-190. doi:10.1056/nejmc2007617

5. Castagnoli R, Votto M, Licari A, et al. Severe acute respiratory syndrome coronavirus 2 (SARS-CoV-2) infection in children and adolescents: a systematic review. JAMA Pediatr. 2020;174(9):882-889.

6. Belhadjer Z, Méot M, Bajolle F, et al. Acute heart failure in multisystem inflammatory syndrome in children in the context of global SARS-CoV-2 pandemic. Circulation. 2020;142(5):429-436.

7. Licciardi F, Pruccoli G, Denina M, et al. SARS-CoV-2-Induced Kawasaki-Like Hyperinflammatory Syndrome: A Novel COVID Phenotype in Children. Pediatrics. May 2020:[published online ahead of print, 2020 May 21]. doi:10.1542/peds.2020-1711

8. Riphagen S, Gomez X, Gonzalez-Martinez C, Wilkinson N, Theocharis P. Hyperinflammatory shock in children during COVID-19 pandemic. Lancet. 2020;395(10237):1607-1608.

9. Verdoni L, Mazza A, Gervasoni A, et al. An outbreak of severe Kawasaki-like disease at the Italian epicentre of the SARS-CoV-2 epidemic: an observational cohort study. Lancet. 2020;395(10239):1771-1778. doi:10.1016/S0140-

6736(20)31103-X

10. Brogan P, Burns JC, Cornish J, et al. Lifetime cardiovascular management of patients with previous Kawasaki disease. Heart. 2020;106(6):411-420.

11. Dionne A, Dahdah N. Myocarditis and Kawasaki disease. Int J Rheum Dis. 2018;21(1):45-49.

12. Piram M, Bello MD, Tellier S, et al. Defining the risk of first intravenous immunoglobulin unresponsiveness in non- 
Asian patients with Kawasaki disease. Sci Rep. 2020;10(1):1-10.

13. Kumrah R, Vignesh P, Rawat A, Singh S. Immunogenetics of Kawasaki disease. Clin Rev Allergy Immunol. 2020;59(1):122-139. doi:10.1007/s12016-020-08783-9

14. Nagelkerke SQ, Tacke CE, Breunis WB, et al. Extensive ethnic variation and linkage disequilibrium at the FCGR2/3 locus: different genetic associations revealed in Kawasaki disease. Front Immunol. 2019;10:185.

15. Elakabawi K, Lin J, Jiao F, Guo N, Yuan Z. Kawasaki Disease: Global Burden and Genetic Background. Cardiol Res. 2020;11(1):9-14. doi:10.14740/cr993

16. Kapustová L, Petrovičová O, Jeselvnák M. Covid-19 a jeho špecifiká pre detsk\{ły\} vek. Pediatria (Santiago). 2020;83:2.

17. Pouletty $\mathrm{M}$, Borocco $\mathrm{C}$, Ouldali N, et al. Paediatric multisystem inflammatory syndrome temporally associated with SARS-CoV-2 mimicking Kawasaki disease (Kawa-COVID-19): a multicentre cohort. Ann Rheum Dis. 2020;79(8):9991006.

18. McCrindle BW, Rowley AH, Newburger JW, et al. Diagnosis, treatment, and long-term management of Kawasaki disease: a scientific statement for health professionals from the American Heart Association. Circulation. 2017;135(17):e927--e999.

19. Olekšák F, Šparcová A. Covid-19 a kardiovaskulárne choroby. Pediatria (Santiago). 2020;93:2.

20. Yang $\mathrm{X}, \mathrm{Yu} \mathrm{Y}, \mathrm{Xu}$ J, et al. Clinical course and outcomes of critically ill patients with SARS-CoV-2 pneumonia in Wuhan, China: a single-centered, retrospective, observational study. Lancet Respir Med. 2020;8(5):475-481. doi:10.1016/S2213-2600(20)30079-5

21. Tirelli F, Marrani E, Giani T, Cimaz R. One year in review: Kawasaki disease. Curr Opin Rheumatol. 2020;32(1):1520. doi:10.1097/BOR.0000000000000671

22. Doung TT, Silverman ED, Bissessar M V., Yeung RSM. Superantigenic activity is responsible for induction of coronary arteritis in mice: An animal model of Kawasaki disease. Int Immunol. 2003;15(1):79-89. doi:10.1093/intimm/dxg007

23. Yeung RSM. Kawasaki disease: Update on pathogenesis. Curr Opin Rheumatol. 2010;22(5):551-560. doi:10.1097/BOR.0b013e32833cf051

24. Dietz SM, van Stijn D, Burgner D, et al. Dissecting Kawasaki disease: a state-of-the-art review. Eur J Pediatr. 2017;176(8):995-1009. doi:10.1007/s00431-017-2937-5

25. Altable M, de la Serna JM. Neuroinflammation links COVID-19 and fragile X syndrome: Role of MMP-9, IGF-1, IL-10, metformin, statins and curcumin. Qeios. 2021;doi:10.32388/KO4C77.2

26. Shulman ST, Rowley AH. Kawasaki disease: Insights into pathogenesis and approaches to treatment. Nat Rev Rheumatol. 2015;11(8):475-482. doi:10.1038/nrrheum.2015.54

27. Ballester J, Borràs S, Curcoll R, et al. On the interpretation of the atmospheric mechanism transporting the environmental trigger of Kawasaki disease. PLoS One. 2019;14(12). doi:10.1371/journal.pone.0226402

28. Rodó X, Curcoll R, Robinson M, et al. Tropospheric winds from northeastern China carry the etiologic agent of Kawasaki disease from its source to Japan. Proc Natl Acad Sci U S A. 2014;111(22):7952-7957.

doi:10.1073/pnas.1400380111 
29. Xie X, Shi X, Liu M. The Roles of Genetic Factors in Kawasaki Disease: A Systematic Review and Meta-analysis of Genetic Association Studies. Pediatr Cardiol. 2018;39(2):207-225. doi:10.1007/s00246-017-1760-0

30. Demirkaya E, Arici ZS, Romano M, Berard RA, Aksentijevich I. Current State of Precision Medicine in Primary Systemic Vasculitides. Front Immunol. 2019;10. doi:10.3389/fimmu.2019.02813

31. Schulte DJ, Yilmaz A, Shimada K, et al. Involvement of Innate and Adaptive Immunity in a Murine Model of Coronary Arteritis Mimicking Kawasaki Disease. J Immunol. 2009;183(8):5311-5318. doi:10.4049/jimmunol.0901395

32. Jones VG, Mills M, Suarez D, et al. COVID-19 and Kawasaki Disease: Novel Virus and Novel Case. Hosp Pediatr. 2020;10(6). doi:10.1542/hpeds.2020-0123

33. Noval Rivas M, Wakita D, Franklin MK, et al. Intestinal Permeability and IgA Provoke Immune Vasculitis Linked to Cardiovascular Inflammation. Immunity. 2019;51(3):508-521.e6. doi:10.1016/j.immuni.2019.05.021

34. Wang Y, Qian SY, Yuan Y, et al. Do cytokines correlate with refractory Kawasaki disease in children? Clin Chim Acta. 2020;506:222-227. doi:10.1016/j.cca.2020.03.014

35. $\mathrm{Xu} \mathrm{Y,} \mathrm{Li} \mathrm{X,} \mathrm{Zhu} \mathrm{B,} \mathrm{et} \mathrm{al.} \mathrm{Characteristics} \mathrm{of} \mathrm{pediatric} \mathrm{SARS-CoV-2} \mathrm{infection} \mathrm{and} \mathrm{potential} \mathrm{evidence} \mathrm{for} \mathrm{persistent} \mathrm{fecal}$ viral shedding. Nat Med. 2020;26(4):502-505. doi:10.1038/s41591-020-0817-4

36. Zheng S, Fan J, Yu F, et al. Viral load dynamics and disease severity in patients infected with SARS-CoV-2 in Zhejiang province, China, January-March 2020: Retrospective cohort study. BMJ. 2020;369. doi:10.1136/bmj.m1443 37. Varga Z, Flammer AJ, Steiger P, et al. Endothelial cell infection and endotheliitis in COVID-19. Lancet. 2020;395(10234):1417-1418. doi:10.1016/S0140-6736(20)30937-5

38. Armaroli G, Verweyen E, Pretzer C, et al. Monocyte-Derived Interleukin-1 $\beta$ As the Driver of S100A12-Induced Sterile Inflammatory Activation of Human Coronary Artery Endothelial Cells: Implications for the Pathogenesis of Kawasaki Disease. Arthritis Rheumatol. 2019;71(5):792-804. doi:10.1002/art.40784

39. Nandi A, Pal P, Basu S. A comparison of serum IL6 and CRP levels with respect to coronary changes and treatment response in Kawasaki disease patients: a prospective study. Rheumatol Int. 2019;39(10):1797-1801. doi:10.1007/s00296019-04375-9

40. Kone-Paut I, Cimaz R, Herberg J, et al. The use of interleukin 1 receptor antagonist (anakinra) in Kawasaki disease: A retrospective cases series. Autoimmun Rev. 2018;17(8):768-774. doi:10.1016/j.autrev.2018.01.024

41. Burns JC, Koné-Paut I, Kuijpers T, Shimizu C, Tremoulet A, Arditi M. Review: Found in Translation: International Initiatives Pursuing Interleukin-1 Blockade for Treatment of Acute Kawasaki Disease. Arthritis Rheumatol. 2017;69(2):268276. doi:10.1002/art.39975

42. Hadjadj J, Yatim N, Barnabei L, et al. Impaired type I interferon activity and inflammatory responses in severe COVID-19 patients. Science (80- ). 2020;369(6504):718-724. doi:10.1126/science.abc6027

43. Trouillet-Assant S, Viel S, Gaymard A, et al. Type I IFN immunoprofiling in COVID-19 patients. J Allergy Clin Immunol. 2020;146(1):206-208.

44. Dobrovanov O, Kralinsky K, Krcmery V. Zaciatok sezony respiracnych infekcii a inicialna antibioticka terapia. Pediatr. 2018;13(5):245-250.

45. Králinskl’y K, Kmečová L, Dobrovanov O. Nová éra antimikrobiálnej terapie. Abstrakty prednášok. 50. celoslovenská pediatrická konferencia s medzinárodnou účast’ou Galandové dni. Pediatr. 2018:S3. 
46. Singhal T. A Review of Coronavirus Disease-2019 (COVID-19). Indian J Pediatr. 2020;87(4):281-286. doi:10.1007/s12098-020-03263-6.

47. Agarwal KM, Sharma P, Bhatia D, Mishra A. Concept Design of the Physical Structure for ICU Ventilators for COVID19 Pandemic. Sensors International. 2021;100092.

48. Cheung EW, Zachariah P, Gorelik M, Boneparth A, Kernie SG, Orange JS, et al. Multisystem inflammatory syndrome related to COVID-19 in previously healthy children and adolescents in New York City. JAMA. 2020 Jul;324(3):294-6. 49. Yi Y, Lagniton PNP, Ye S, Li E, Xu RH. COVID-19: what has been learned and to be learned about the novel coronavirus disease. Int J Biol Sci 2020; 16(10):1753-1766.

50. Abraham D, Kalyanasundaram S, Krishnamurthy K. Refractory Kawasaki Disease-a Challenge for the Pediatrician. SN Compr. Clin. Med.2021; 3, 855-860 .

51. Nalbandian A, Sehgal K, Gupta A. et al. Post-acute COVID-19 syndrome. Nat Med .2021, 27, 601-615. 